\title{
The Relation between the Permeability of Structured Dispersions and the Viscoelastic Properties of the Dispersed Phase
}

\author{
H. KAMPHUIS, R. J. J. JONGSCHAAP, AND P. BOUTER \\ Rheology Group, Department of Applied Physics, Twente University of Technology, Enschede, The Netherlands
}

Received July 18, 1983; accepted September 22, 1983

\begin{abstract}
It is shown that not only the average particle size and compression modulus, but also the viscoelastic properties of the network affect the rate of liquid removal out of a concentrated disperse system that is unilaterally compressed. A Maxwell-like constitutive equation is introduced to express these viscoelastic properties. The rate of liquid removal is calculated for a set of values of the elastic modulus and the time constant that appear in the constitutive equation. It is shown that permeability measurements on concentrated fat dispersions can be interpreted in terms of network properties also when the network is viscoelastic, i.e., it is assumed that processes take place inside the network that involve energy dissipation.
\end{abstract}

\section{INTRODUCTION}

Permeability measurements have been found to be successful for determining the average particle size in systems that consist of a compressible solid matrix, which may be considered as a network, the pores of which are filled with a liquid (1). These systems will be referred to as structured disperse systems. Both the specific surface and the elastic modulus of the solid phase affect the liquid-flow rate out of a structured disperse system that is unilaterally compressed. When combining permeability measurements for two values of the applied load, the specific surface of the solid can be determined. The method assumes the solidstate interparticle bonds to be permanent within the time scale of the experiment. The theory that underlies the method confirms the square-root law of Terzaghi (2), which states that the total amount of liquid that is squeezed out of the disperse system is proportional to the square root of the time provided that the load is small enough.

Experimental results obtained by means of the method described above are connected with both the average particle size and the network-compression modulus. A method was introduced by Buscall (3) that gives the value of the network-compression modulus itself in a more straightforward manner. The disperse system experiences a centrifugal-force field. The network compression modulus is then found from the equilibrium solid-phase sedimentation volume. Although the method contains elements of permeability measurements, it does not involve the solid's specific surface. This is because only the equilibrium sedimentation volume is taken into account when there is no liquid flow left with respect to the solid matrix. The method assumes the network to behave as a purely elastic material once the steady-state situation has been reached. In permeametric as well as centrifugal experiments both elastic and network-shape properties affect the rate at which the liquid penetrates the system. However, network elastic and shape properties can be revealed separately, either by performing at least two permeability measurements and combining them in a proper way (1), or by considering centrifugal experiments at time scales where effects due to liquid flow may be assumed to be negligible (3).

When the network chains assume a finite life-time and may break within the time scale of the experiment, or when there is some rearrangement of network particles with respect 
to each other, the normal stress that has accumulated in the network may relax even at a constant network deformation, i.e., the network may behave like a viscoelastic material rather than as a purely elastic one. In this case, the viscoelastic-network properties may affect the apparent permeability or compressibility of such systems. This infers that, in principle, both types of experiments also reveal information about the viscoelastic properties of the network phase.

The present paper describes the way in which viscoelastic-network properties may affect permeability measurements. It will be shown that deviations from Terzaghi's squareroot law, which have been experimentally observed, can be explained in this way. This provides us with a tool for estimating the relaxation time that characterizes the processes that take place in the solid phase which cause stress relaxation. The same mechanisms cause the complex shear modulus to assume different values as a function of the radial frequency. The linear viscoelastic shear behavior and the permeability of such systems are interrelated through the model presented in this paper. Use is made of the relationship between the shear modulus of the system as a whole and the compression modulus of the network that was found by Buscall (3).

In order to investigate the model experimentally, concentrated fat dispersions have been analyzed in terms of their linear viscoelastic behavior in shear experiments and their permeability.

\section{THEORY}

The analysis presented in this paper parallels the theory of De Jager et al. (1) as far as elasticnetwork properties are concerned. The network structure and the notations that are used to describe its state of deformation are presented in Fig. 1.

A constant load $P$ is applied at time $t=0$. The liquid will then start to flow through the filter, which is impermeable to network par-

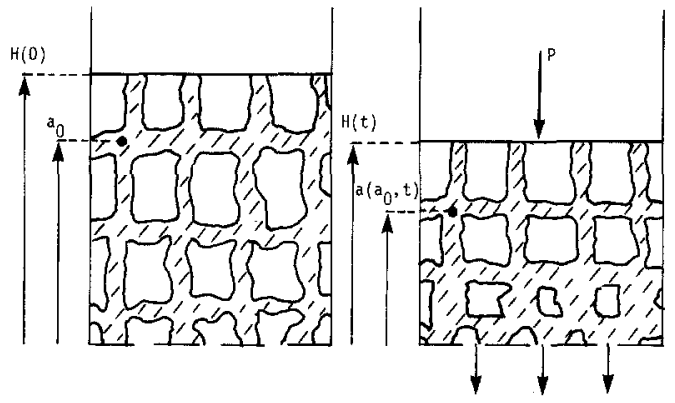

FIG. 1. Network compressed by means of a load $P$. The bottom filter transmits only the liquid.

ticles. A Lagrangian coordinate $a_{0}$ is introduced to describe the network deformation as a function of time. The component of the network-deformation gradient in the direction of the flow reads

$$
F\left(a_{0}, t\right) \equiv \frac{\partial a\left(a_{0}, t\right)}{\partial a_{0}} .
$$

The deformation rate of the network as a function of the Lagrangian coordinate $a_{0}$ and the time $t$ was found to read for $a_{0}>0$ (Eq. (3.14) in ref.1):

$$
\begin{aligned}
& \frac{\partial}{\partial t} F\left(a_{0}, t\right) \\
& \quad=-\frac{\partial}{\partial a_{0}}\left\{\frac{K\left(\epsilon\left(F\left(a_{0}, t\right)\right)\right)}{\mu F\left(a_{0}, t\right)} \frac{\partial p\left(a_{0}, t\right)}{\partial a_{0}}\right\},
\end{aligned}
$$

in which $\epsilon_{0}$ is the initial value of $\epsilon\left(a_{0}, t\right)$, being the volume fraction that is occupied by the liquid, $\mu$ is the liquid viscosity, $p\left(a_{0}, t\right)$ is the effective normal stress as far as it results from network deformation, and $K(F)$ is the permeability of the system for which the KozenyCarman relation is assumed to apply:

$$
K(F)=\frac{1}{5 S^{2}} \frac{\epsilon^{3}(F)}{(1-\epsilon(F))^{2}} .
$$

$S$ is the specific surface area of the solid. Other expressions have been introduced in literature that connect the permeability of porous media and the structural properties of the three-dimensional networks. The connectivity in pore 
space is a central theme in "cutting and joining models" that express the permeability of porous media in terms of the volume fraction of liquid and the distribution of pore radii (4-6). Adler et al. (7) calculated the permeability of a fixed swarm of permeable spheres by means of a cell model.

The system porosity is related to the network strain as

$$
\epsilon\left(a_{0}, t\right)=1-\frac{1-\epsilon_{0}}{F\left(a_{0}, t\right)}
$$

(see ref. 1).

The amount of liquid that is transmitted by a unit area of the filter reads

$$
H(0)-H(t)=\int_{0}^{H(0)}\left(1-F\left(a_{0}, t\right)\right) d a_{0} .
$$

A network with viscoelastic properties. Even in a state of rest, stress relaxation may not be excluded, e.g., if the bond energy is not high compared to the unit energy $k T$, connected with thermal fluctuations. Normal stress relaxation will cause the strain that is accumulated in the interparticle links to be not fully recoverable. Besides, it causes the network to consolidate such that the normal stress due to network compression together with the isotropic liquid pressure equal the applied load. This may subsequently affect the amount of liquid that flows through the filter. For a quantitative interpretation of this effect, Eq.

(2) needs an additional expression that accounts for normal stress relaxation. The effective normal stress, $p$, is related to the average stress that is transmitted through a unit surface of network-phase material, $\sigma$, by

$$
p\left(a_{0}, t\right)=-\left(1-\epsilon\left(a_{0}, t\right)\right) \sigma\left(a_{0}, t\right) .
$$

Differentiation with respect to the time yields

$$
\begin{aligned}
\frac{\partial p\left(a_{0}, t\right)}{\partial t}=-\left(1-\epsilon\left(a_{0}, t\right)\right) \frac{\partial \sigma\left(a_{0}, t\right)}{\partial t} \\
+\sigma\left(a_{0}, t\right) \frac{\partial \epsilon\left(a_{0}, t\right)}{\partial t} .
\end{aligned}
$$

Combination of Eqs. [4], [6], and [7] gives

$$
\begin{aligned}
\frac{\partial p\left(a_{0}, t\right)}{\partial t}=-\left(\frac{1-\epsilon_{0}}{F\left(a_{0}, t\right)} \frac{\partial \sigma\left(a_{0}, t\right)}{\partial t}\right. & \\
& \left.+\frac{p\left(a_{0}, t\right)}{F\left(a_{0}, t\right)} \frac{\partial F\left(a_{0}, t\right)}{\partial t}\right) .
\end{aligned}
$$

It is seen that the effective normal stress that results from network compression may change due to stress relaxation that takes place inside the dispersed phase and due to a change of the volume fraction of dispersed material.

In order to describe the stress relaxation in the dispersed phase, we use a simple equation of linear viscoelastic behavior with one relaxation time and one modulus. This expression, of the so-called Maxwell type (8), reads:

$$
\frac{\partial \sigma\left(a_{0}, t\right)}{\partial t}=-\frac{\sigma\left(a_{0}, t\right)}{\lambda}+\kappa \frac{\frac{\partial}{\partial t} F\left(a_{0}, t\right)}{F\left(a_{0}, t\right)},
$$

in which $\kappa$ has the character of the elastic modulus and $\lambda$ is a relaxation time. The term $(\partial / \partial t) F\left(a_{0}, t\right) / F\left(a_{0}, t\right)$ stands for the component in the direction of the flow of the average rateof-strain tensor, which is defined in the solid matrix.

On substituting Eq. [9] into Eq. [8] and using Eqs. [4] and [6], we obtain

$$
\begin{gathered}
\frac{\partial p\left(a_{0}, t\right)}{\partial t}=-\left\{\frac{p\left(a_{0}, t\right)}{\lambda}+\left(\left(1-\epsilon_{0}\right) \kappa\right.\right. \\
\left.\left.+p\left(a_{0}, t\right) F\left(a_{0}, t\right)\right) \frac{\frac{\partial}{\partial t} F\left(a_{0}, t\right)}{F^{2}\left(a_{0}, t\right)}\right\} .
\end{gathered}
$$

Equations [2] and [10] give the network deformation as a function of the Lagrangian coordinate and time. The total liquid volume per unit area extracted from the disperse system follows from Eq. [5].

Initial and boundary conditions. The initial and boundary conditions required to solve Eqs. [2] and [10] have been reported elsewhere (1) and are repeated here for the sake of convenience: 


$$
\begin{aligned}
& F\left(a_{0}, t\right)=1 \quad \text { for } \quad t=0 \quad \text { and } \quad 0 \leqslant a_{0} \leqslant \mathbf{H}(0) \\
& \frac{\partial}{\partial a_{0}} F\left(a_{0}, t\right)=0 \quad \text { for } \quad t \geqslant 0 \quad \text { and } \quad a_{0}=\mathrm{H}(0) \\
& p\left(a_{0}, t\right)=P \quad \text { for } \quad t>0 \quad \text { and } \quad a_{0}=0 \\
& =0 \text { for } t=0 \text { and } 0 \leqslant a_{0} \leqslant \mathrm{H}(0) \text {. }
\end{aligned}
$$

In the case that $a_{0}=0$, during the very short time interval that the load establishes itself, both time derivatives that appear in Eq. [10] assume large values compared to the relaxation term. Thus, we may write for short times

$$
\begin{aligned}
& \lim _{t \perp 0} \frac{d p(0, t)}{d F(0, t)} \\
& \quad=-\lim _{t \downarrow 0}\left(\frac{\left(1-\epsilon_{0}\right) \kappa}{F^{2}(0, t)}+\frac{p(0, t)}{F(0, t)}\right) .
\end{aligned}
$$

Equation [14], when supplied with boundary condition [13], can be solved by means of the method of variation of constants. We find

$$
\frac{\ln F(0, t)}{F(0, t)}=\frac{P}{\left(1-\epsilon_{0}\right) K}(t \downarrow 0) \text {. }
$$

The solid matrix at the bottom of the sample holder is compressed more and more as long as stress relaxation takes place in the solid matrix. The rate of compression follows from Eq. [10] when supplied with condition [13]:

$$
\frac{\partial F(0, t)}{\partial t}=-\frac{P}{\lambda}\left(\frac{F^{2}(0, t)}{\left(1-\epsilon_{0}\right) \kappa+P F(0, t)}\right) \text {. }
$$

It is seen that no further network compression takes place in the limit $\lambda \rightarrow \infty$, i.e., in the case that the network behaves elastically. It should be noticed that Eq. [16] can only give an approximate description of the deformation process that takes place in the bottom layer. When $F(0, t)$ approaches $1-\epsilon_{0}$, all volume is occupied by the solid phase, which causes the stress to rise to infinity when the solid particles themselves are incompressible. Equations [9], [15], and [16] do not account for this effect.

\section{MODEL PREDICTIONS}

The aim of this section is to illustrate in what manner the viscoelastic properties of the network affect permeametric experiments. The quantity of prime interest to the investigator is the liquid volume that has been extracted since the start of the experiment (see Eq. [5]). This quantity is found from Eqs. [2] and [10], together with initial and boundary conditions [11], [12], [15], and [16], by means of numerical integration. The square-root law motivates to replace $t$ in the fundamental equations by $y \equiv \sqrt{t}$ as the independent variable. The extracted volume is thus calculated as a function of $y$. Any deviation from linearity then indicates that there is no agreement with Terzaghi's law.

Material properties of the fat dispersion extensively described under Experiments (see Table I) have been used here to demonstrate some typical aspects of the model. Flow calculations have been carried out for two extreme values of the applied load $P$. They explain in what way the viscoelastic properties of the network and the permeability of the sample as a whole are interrelated.

In either of Figs. 2 and 3 the extracted volume per unit area has been plotted as a function of time. The influence of network viscoelasticity is visualized by carrying out calculations for various values of $\lambda$. In Fig. 2 the applied load is relatively low with respect to the network-compression modulus, whereas a relatively high value was used in Fig. 3. It can be seen that for $\lambda \rightarrow \infty$ Terzaghi's squareroot law is satisfied. In addition, the approx- 


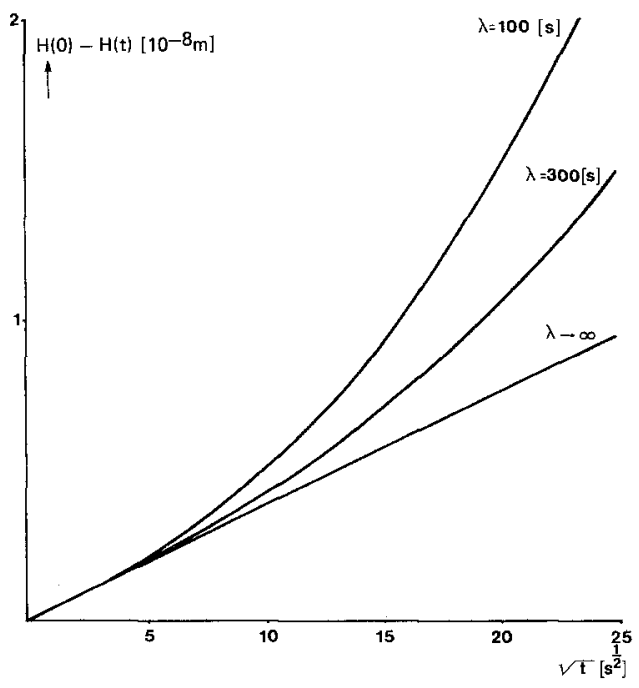

FIG. 2. Liquid volume per unit area squeezed out of a network that is unilaterally compressed, according to Eqs. [2] and [10]. Applied load $P=1\left(\mathrm{~N} \mathrm{~m}^{-2}\right)$, the relaxation time $\lambda$ is varied so as to demonstrate its effect on the permeation process. See Table I for other parameter values.

imate solution derived by De Jager et al. (1) coincides exactly with the straight line in Fig. 2 and approximately with the one in Fig. 3. In the case that $P \ll\left(1-\epsilon_{0}\right) \kappa$, this approximate solution can be written as

$H(t)-H(0)$

$$
=2 P \sqrt{\frac{\epsilon_{0}^{3}}{5 \pi \mu S^{2} \kappa\left(1-\epsilon_{0}\right)^{3}}} \sqrt{t},
$$

as can be seen from ref. (1) and Eq. [15].

It is seen that the way in which the introduction of a finite relaxation time $\lambda$ affects the permeation process does not only depend on the value of $\lambda$ itself but also on the value of $P$. The ratio of special interest is $P /\left(1-\epsilon_{0}\right) \kappa$ (Eq. [15]). The effect that the liquid flow rate has either increased of decreased when $\lambda$ assumes a finite value is explained by the generalized diffusion coefficient in Eq. [2]:

$$
\begin{aligned}
\frac{K(F)}{\mu F}=\frac{1}{5 \mu S^{2}}\left(\frac{-3}{1-\epsilon_{0}}\right. & +\frac{F}{\left(1-\epsilon_{0}\right)^{2}} \\
& \left.+\frac{3}{F}-\frac{1-\epsilon_{0}}{F^{2}}\right) .
\end{aligned}
$$

Let us assume that $P /\left(\left(1-\epsilon_{0}\right) \kappa\right)$ is small compared to unity. This means that the load will only cause a minor compression of the network. If we write $F\left(a_{0}, t\right) \equiv 1-\Delta F\left(a_{0}, t\right)$, with $\Delta F\left(a_{0}, t\right) \ll 1$, Eq. [18] equals

$$
\frac{1}{5 \mu S^{2}}\left\{\frac{\epsilon_{0}^{3}}{\left(1-\epsilon_{0}\right)^{2}}+\Delta F\left(1+2 \epsilon_{0}-\frac{1}{\left(1-\epsilon_{0}\right)^{2}}\right)\right\} \text {. }
$$

It is seen that network-stress relaxation will cause only a relatively small decrease in the value of the generalized diffusion coefficient. On the other hand, Eqs. [8] and [9] show that network-stress relaxation will cause the network-pressure gradient to be larger than when no such relaxation occurs. The quantity that promotes liquid flow, i.e., the liquid-pressure gradient $\left(\equiv-\partial p\left(a_{0}, t\right) / \partial a_{0}\right)$ then completely overwhelms the permeability decrease resulting from a decrease in porosity. It can be concluded, in this case, that the viscoelastic effects enhance the liquid flow rate out of the system.

Let us now assume that $P /\left(\left(1-\epsilon_{0}\right) \kappa\right)$ is large compared to unity. The deformation gradient for $a_{0}=0$ is then far less than unity, i.e., it will approach $1-\epsilon_{0}$ (see Eq. [2]). Equation [18] will then tend to zero and cause the diffusion coefficient in Eq. [2] to become very

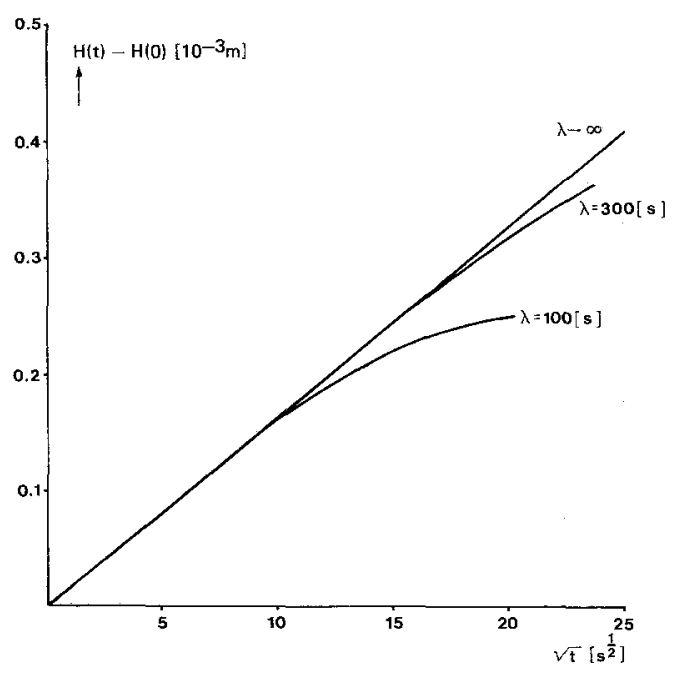

FIG. 3. The same as Fig. 2. The applied load $P$ was taken as $1 \times 10^{5}\left(\mathrm{~N} \mathrm{~m}^{-2}\right)$ for this calculation. 
small. In other words, the porosity at the bottom of the sample is much smaller than it was before load pressure was applied. It can easily be imagined that in such a situation networkstress relaxation causes a further decrease in permeability of the bottom layer of the sample. Liquid flow is then considerably delayed, since this effect cannot be completely compensated by the increase in the liquid-pressure gradient. It can be concluded that in this case the viscoelastic effects will cause a decrease in permeability of the sample as a whole.

\section{EXPERIMENTS}

Permeametric measurements were performed on a 20 wpct dispersion of glyceroltristearate crystals in paraffin oil at $22.7^{\circ} \mathrm{C}$. The porosity $\epsilon_{0}$ is about 0.825 , whereas the viscosity of the liquid phase is about 0.127 [N $\left.\mathrm{m}^{-2} \mathrm{~s}\right]$. The storage modulus $G^{\prime}$ was measured in cone-and-plate configuration on a Rheometrics mechanical spectrometer and was found to be about $4 \times 10^{4}\left(\mathrm{~N} \mathrm{~m}^{-2}\right)$ at a radial frequency of $1\left(\mathrm{rad} \mathrm{sec}^{-1}\right)$. It should be noticed that the sample was not given time to undergo any time-hardening, i.e., the storage modulus is seen to rise when the sample is preserved at rest. By taking into account the liquid-volume fraction (Eq. [4]) and using Buscall's relation $K^{\prime}=5 / 3 G^{\prime}$, in which $K^{\prime}$ is the apparent compression modulus of the dispersed phase as a whole and $G^{\prime}$ is its shear modulus; the elastic modulus $\kappa$ of the network is estimated to be about $K^{\prime} /\left(1-\epsilon_{0}\right) \simeq 4 \times 10^{5}\left(\mathrm{~N} \mathrm{~m}^{-2}\right)$ $(1,3)$. The specific surface of the crystals is estimated to be about $0.6 \times 10^{8}\left(\mathrm{~m}^{-1}\right)(1,9)$ (see Table I).

The amount of liquid squeezed out per unit area was measured as a function of the square root of the time after a constant load was applied at time $t=0$ (see Fig. 4).

It can be seen that there is a good agreement with Terzaghi's law, apart from some irregular effect at the beginning of the experiment. It is important to know whether this phenomenon can be ascribed to network normal stress relaxation, and, therefore, to find out whether

\section{TABLE I}

Properties of the 20 wpct Dispersion of Glyceroltristearate Crystals in Paraffin Oil Used for Permeatric Measurements

\begin{tabular}{lll}
\hline$\epsilon_{0}$ & & 0.825 \\
$\mu$ & $\left(\mathrm{N} \mathrm{m}^{-2} \mathrm{~s}\right)$ & 0.127 \\
$\kappa$ & $\left(10^{5} \mathrm{~N} \mathrm{~m}^{-2}\right)$ & 4 \\
$S$ & $\left(10^{8} \mathrm{~m}^{-1}\right)$ & 0.6 \\
$H(0)$ & $\left(10^{-3} \mathrm{~m}\right)$ & 5.6 \\
\hline
\end{tabular}

a nonfinite value of $\lambda$ will cause either an increase or a decrease in the apparent permeability. Model calculations have been carried out by making use of the model parameters listed in Table I and by substitution of proper values of the applied stress and the relaxation time that gives the best overall fit (see Fig. 4).

It is seen that network-stress relaxation will cause an increase in the liquid flow rate at the beginning, whereas in the course of the experiments the bottom layer appears to consolidate, which causes the response to flatten to such an extent that a new region is found where Terzaghi's law seems to be obeyed in spite of stress relaxation. It is seen that both effects, demonstrated in Figs. 2 and 3, almost cancel each other out in course of time in case the applied stress attains a value somewhere in the region of 0.1 to $0.3 \times 10^{5}\left(\mathrm{~N} \mathrm{~m}^{-2}\right)$. Thus, the solid phase need not necessarily behave purely elastically for complying with Terzaghi's law within a reasonable approximation.

Figure 5 illustrates the microscopic-deformation gradient that corresponds to the calculations made to fit the experimental results (Fig. 4). It is seen that the shape of the curves is substantially affected by the value of $\lambda$. The network in the bottom layer is seen to be considerably more deformed when $\lambda=100$ (sec) than in the case that $\lambda \rightarrow \infty$. However, the opposite is seen to be true for $a_{0}>0.7\left(10^{-3}\right.$ $\mathrm{m})$. Equation [5] shows that the areas below the curves correspond to the liquid volume that has been squeezed out. It is seen that these areas need not be too much different 


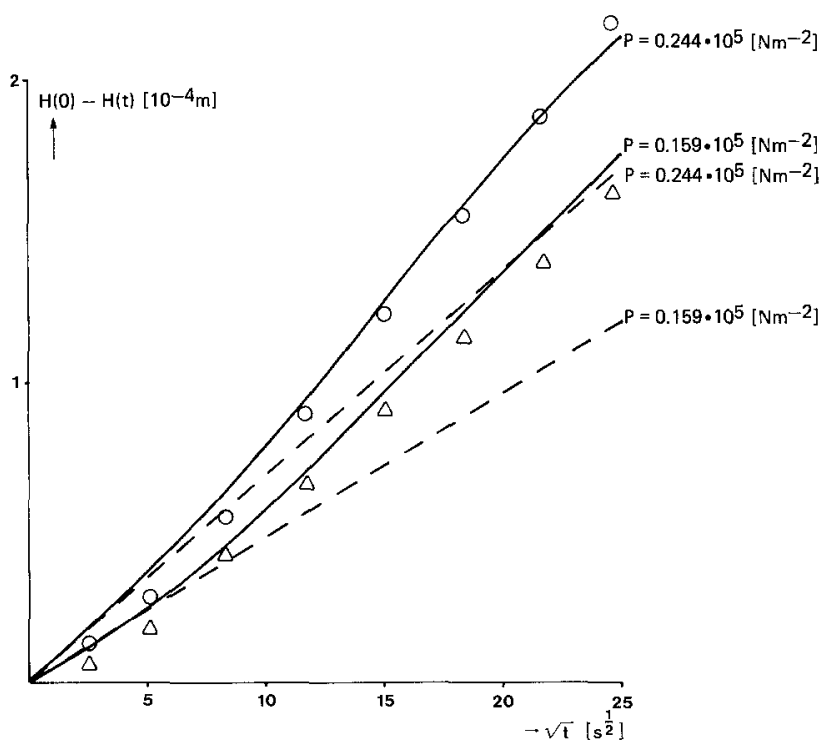

FiG. 4. Liquid volume per unit area squeezed out of a $20 \mathrm{wpct}$ dispersion of glyceroltristearate crystals in paraffin oil, being crystallized at $25^{\circ} \mathrm{C}$. The symbols denote experimental results: $\triangle, P=0.159 \times 10^{5}$ $\left(\mathrm{N} \mathrm{m}^{-2}\right) ; O, P=0.244 \times 10^{5}\left(\mathrm{~N} \mathrm{~m}^{-2}\right) ;(---)$, model calculation for $\lambda \rightarrow \infty ;(-)$, model calculation for $\lambda=100$ (s). See Table I for other parameter values.

from each other although the shapes of the curves are far different from each other. It can be expected from linear viscoelastic data that considerable stress relaxation will take place within the time scale of the experiments. Figures 4 and 5 illustrate why this effect does not prevent Terzaghi's square-root law from being approximately valid.

It should be remarked at this point that in permeatric experiments on 5 wpct fat-crystal dispersions no linear relationship was found to exist between the amount of liquid squeezed out and the square root of time. Instead, the amount of liquid squeezed out seemed to increase linearly with time. The shape of the curves shown in Fig. 2 correspond to these measurements rather than the shape predicted by Terzaghi's law. Network-stress relaxation could be the microscopic process that is responsible for this type of behavior.

\section{CONCLUSIONS}

Network-stress relaxation may cause permeability measurements to deviate from the square-root law proposed by Terzaghi. The way in which these effects will change the apparent permeability is seen to be closely connected with system properties, i.e., the network-elastic modulus and the liquid-volume fraction on the one hand, and experimental

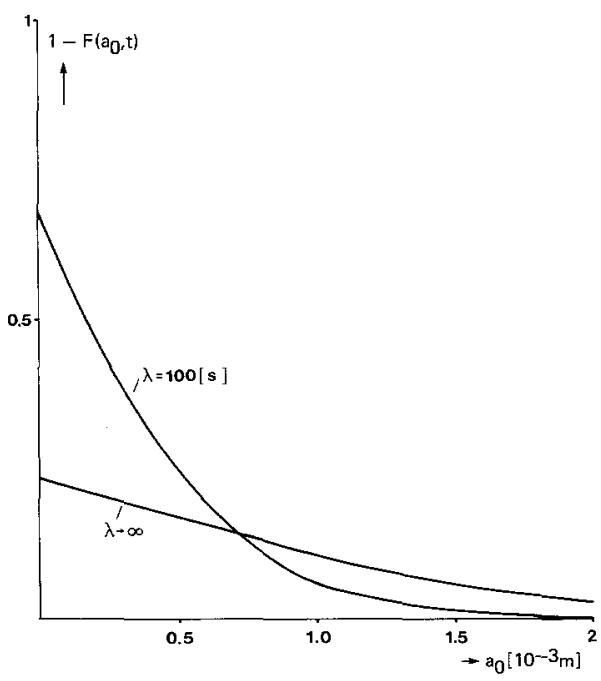

FIG. 5. Deformation gradient in a disperse system 625 sec after a constant load $P=0.244 \times 10^{5}\left(\mathrm{~N} \mathrm{~m}^{-2}\right)$ was applied, according to Eqs. [2] and [10] (see Table I). 
conditions, i.e., the applied load on the other. The model predicts, for the fat dispersion used for experiments, that network-stress relaxation, which is likely to occur, will cause the liquid flow rate to increase somewhat. However, Terzaghi's law is not expected to be disobeyed too much. This expectation is supported by experiments on this system.

\section{APPENDIX 1: LIST OF SYMBOLS}

$a, a_{0} \quad$ Place coordinates

$F \quad$ Deformation gradient component

$H \quad$ Height of the sample

$K \quad$ Permeability

$P \quad$ Applied load

$p \quad$ Effective normal pressure

$S \quad$ Specific surface

$t \quad$ Time

$\epsilon, \epsilon_{0} \quad$ Porosity
Elastic modulus

Relaxation time

Liquid viscosity

Average normal stress in the network

\section{REFERENCES}

1. De Jager, E. M., Van den Tempel, M., and De Bruyne, P., Koninklijke Acad. Wetenschappen B66, 17 (1963).

2. Terzaghi, K., in "Theoretical Soil Mechanics," Chs. XII and XIII, 2nd ed. Wiley, New York, 1965.

3. Buscall, R., Colloid Surf. 5, 269 (1982).

4. Marshall, T. J., Symp. Interact. Fluids Particles, p. 299, London (1962).

5. Millington, R. J., and Quirik, J. P., Trans. Faraday Soc. 57, 1200 (1962).

6. Mahgoub, I., Prost, C., and Dodds, J. A., Powder Technol. 31, 143 (1982).

7. Adler, P. M., Mills, P. M., and Quemada, D. C., AIChE J. 24, 354 (1978).

8. Maxwell, J. C., Phil. Trans. Roy. Soc. A157, 49 (1867).

9. Nederveen, C. J., J. Colloid Sci. 18, 276 (1963). 